\title{
Choice procedures in Pairwise Comparison Multiple-attribute Decision Making Methods
}

\author{
Raymond Bisdorff ${ }^{1}$ and Marc Roubens ${ }^{2}$ \\ 1 Department of Management and Informatics, University Center of Luxembourg, \\ Bisdorff@cu.lu \\ 2 Department of Mathematics, University of Liege, M.Roubens@ulg.ac.be
}

\begin{abstract}
We consider extensions of some classical rational axioms introduced in conventional choice theory to valued preference relations. The concept of kernel is revisited using two ways: one proposes to determine kernels with a degree of qualification and the other presents a fuzzy kernel where every element of the support belongs to the rational choice set with a membership degree. Links between the two approaches is emphasized. We exploit these results in Multiple-attribute Decision Aid to determine the good and bad choices. All the results are valid if the valued preference relations are evaluated on a finite ordinal scale.
\end{abstract}

\section{Introduction}

We consider a pair wise comparison multiple-attribute decision making procedure that assigns to each ordered pair $(x, y), x, y \in A$ (the set of alternatives) a global degree of preference $R(x, y) . R(x, y)$ represents the degree to which $x$ is weakly preferred to $y$.

We suppose that $R(x, y)$ belongs to a finite set $L:\left\{c_{0}, c_{1}, \ldots, c_{m}, \ldots, c_{2 m}\right\}$ that constitutes a $(2 m+1)$-element chain $\left\{c_{0}, c_{1}, \ldots, c_{2 m}\right\} . R(x, y)$ may be understood as the level of credibility that " $a$ is at least as good as $b$ ". The set $L$ is built using the values of $R$ taking into consideration an antitone unary contradiction operator $\neg$ such that $\neg c_{i}=c_{(2 m-i)}$ for $i=0, \ldots, 2 m$.

If $R(x, y)$ is one of the elements of $L$, then automatically $\neg R(x, y)$ belongs to $L$. We call such a relation an $L$-valued binary relation.

We denote $L^{\succ m}:\left\{c_{m+1}, \ldots, c_{2 m}\right\}$ and $L^{\prec m}:\left\{c_{0}, \ldots, c_{m-1}\right\}$.

If $R(x, y) \in L^{\succ m}$, we say that the proposition " $(x, y) \in R$ " is $L$-true. If however $R(x, y) \in L^{\prec m}$, we say that the proposition is $L$-false. If $R(x, y)=c_{m}$, the median level (a fix point of the negation operator) then the proposition " $(x, y) \in R$ " is $L$-undetermined. If $R(a, b)=c_{r}$ and $R(c, d)=c_{s}, c_{r}<c_{s}$, it means that the proposition " $a$ is at least as good as $b$ " is less credible than " $c$ is at least as good as $d "$.

In the classical case where $R$ is a crisp binary relation $(m=2$, and $R(x, y)$ is never rated $c_{1} ; R(x, y)=c_{2}=1$ is denoted $x R y$ and $R(x, y)=c_{0}=0$ corresponds to $\neg x R y$, we define a digraph $G(A, R)$ with vertex set $A$ and arc family $R$. A choice in $G(A, R)$ is a non empty set $Y$ of $A$. 
$R$ can be represented by a Boolean matrix and the choice $Y$ can be defined with the use of a subset characteristic row vector $Y()=$. $(\ldots, Y(x), \ldots, Y(y), \ldots)$ where

$$
Y(x)=\left\{\begin{array}{l}
1 \text { if } x \in Y \\
0 \text { otherwise, }
\end{array} \text { for all } x \in A .\right.
$$

The subset characteristic vector of the successors of the elements of the vertex set $Y:\{x \in A \mid \exists y \in Y, y R x\}$ is denoted $Y \circ R$ and is obtained using the Boolean composition

$$
(Y \circ R)(x)=\vee_{y \neq x}(Y(y) \wedge R(y, x))
$$

where $\vee$ and $\wedge$ represent respectively "disjunction" and "conjunction" for the 2-element Boolean lattice $\mathbf{B}=\{0,1\}$.

The choice $Y$ should satisfy some of the following rationality axioms $(\bar{Y}$ represents the complement of $Y$ in $A$ ):

- Inaccessibility of $Y$ (or GOCHA rule, cf.[5], [10])

$\forall y \in Y, \forall x \in \bar{Y}, \neg x R y$

$\bar{Y} \circ R \subseteq \bar{Y}$, "the successors of $\bar{Y}$ are inside $\bar{Y}$ ".

- Stability of $Y$ (see [9], [11])

$\forall y \in Y, \forall x \in Y, \neg y R x$

$Y \circ R \subseteq \bar{Y}$, "the successors of $Y$ are inside $\bar{Y}$ ".

- Dominance of $Y$ (or external stability, see [9],[11])

$\forall x \in \bar{Y}, \exists y \in Y, y R x$

$\bar{Y} \subseteq Y \circ R$, "the successors of $Y$ contain $\bar{Y}$ ".

- Strong dominance of $Y$ (or GETCHA rule, cf. [5], [10])

$\forall y \in Y, \forall x \in \bar{Y}, y R x \equiv \neg y R^{d} x$

$\left(R^{d}\right.$ is the dual relation, i.e. the transpose of the complement of $R$ )

$\hat{Y} \circ R^{d} \subseteq \bar{Y}$.

The maximal set of all non-dominated alternatives (inaccessibility and stability are satisfied) is called the core of $Y$ and the internally and externally stable set corresponds to the kernel. The GETCHA set is such that the strong dominance rule applies.

No specific property like acyclicity or antisymmetry will be assumed in the sequel. The core guarantees a rather small choice but is often empty. The GETCHA set corresponds to a rather large set and, in this general framework, the kernel (see [5], [8]) seems to be the best compromise. However its existence or uniqueness cannot be guaranteed. . It has been mentioned in [5] that for random graphs - with probability .5 - a kernel almost certainly exists and that in a Moon-Moser graph with $\mathrm{n}$ nodes the number of kernels is around $3^{n / 3}$.

In order to illustrate all these concepts, we consider a small example. 
Example 1 Consider the following example with 8 alternatives: $A$ : $\{a, b, c, d, e, f, g, h\}$. The Boolean matrix $R$ together with the outgoing and ingoing scores $S(+)$ and $S(-)$ are presented in Table 1.

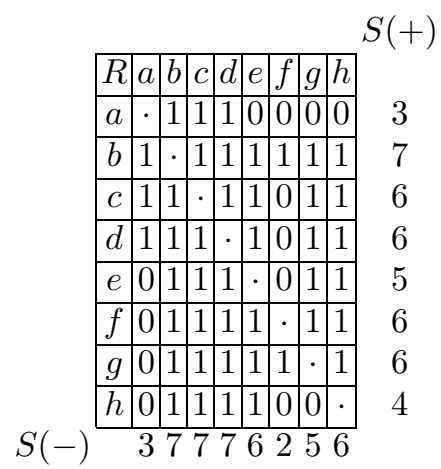

Table 1 : Boolean matrix R and scores

Core (non dominated elements) : empty set.

Kernels (maximal stable and minimal dominant sets) : $\{b\},\{a, f\},\{a, g\}$. Minimal GETCHA sets : $\{b\},\{a, e, f, g, h\}$.

We may define generalizations of the previous crisp concepts in the valued case in two different ways:

(i) Starting from the definition of a rational choice in terms of logical predicates, one might consider that every subset of $A$ is a rational choice with a given qualification and determine those sets with a sufficient degree of qualification.

(ii) One might also extend the algebraic definition of a rational choice. In that case, there is a need to define proper extensions of composition law $\circ$ and inclusion $\subseteq$.

Solutions that correspond to this approach give a fuzzy rational set $\tilde{Y}$, each element of $A$ belonging to $A$ to a certain degree (membership function).

It should be interesting to stress the correspondence between these two approaches. The choice of the operators is closely related to the type of scale that is used to quantify the valued binary relation $R$, i.e. an ordinal scale.

\section{Qualification of crisp kernels in the valued ordinal context}

We now denote $G^{L}=G^{L}(A, R)$ a digraph with vertices set $A$ and a valued arc family that corresponds to the $L$-valued binary relation $R$. This graph is often called outranking graph in the context of multi-attribute decision making.

We define the level of stability qualification of subset $Y$ of $X$ as

$$
\Delta^{s t a}(Y)= \begin{cases}c_{2 m} & \text { if } Y \text { is a singleton, } \\ \min _{y \neq x} \min _{x \neq y}\{\neg R(x, y)\} & \text { otherwise }\end{cases}
$$


and the level of dominance qualification of $Y$ as

$$
\Delta^{d o m}(Y)= \begin{cases}c_{2 m} & \text { if } Y=A, \\ \min _{x \notin Y} \max _{y \in Y} R(y, x) \text { otherwise. }\end{cases}
$$

$Y$ is considered to be an $L$-good choice, i.e $L$-stable and L-dominant, if $\Delta^{s t a}(Y) \in L^{\succ m}$ and $\Delta^{d o m}(Y) \in L^{\succ m}$. Its qualification corresponds to

$$
Q^{\text {good }}(L)=\min \left(\Delta^{s t a}(Y), \Delta^{\text {dom }}(Y)\right) \in L^{\succ m} .
$$

We denote $C^{\text {good }}\left(G^{L}\right)$ the possibly empty set of $L$-good choices in $G^{L}$.

The determination of this set is an NP-complete problem even if, following a result of Kitainik [5], we do not have to enumerate the elements of the power set of $A$ but only have to consider the kernels of the corresponding crisp strict median-level cut relation $R^{\succ m}$ associated to $R$, i.e. $(x, y) \in R^{\succ m}$ if $R(x, y) \in$ $L^{\succ m}$.

As the kernel in $G\left(X, R^{\succ m}\right)$ is by definition a stable and dominant crisp subset of $A$, we consider the possibly empty set of kernels of $G^{\succ m}=G\left(A, R^{\succ m}\right)$ which we denote $C^{\text {good }}\left(G^{\succ m}\right)$.

Kitainik proved that

$$
C^{\text {good }}\left(G^{L}\right) \subseteq C^{\text {good }}\left(G^{\succ m}\right)
$$

The determination of crisp kernels has been extensively described in the literature (see, for example [9]) and the definition of $C^{\text {good }}\left(G^{L}\right)$ is reduced to the enumeration of the elements of $C^{\text {good }}\left(G^{\succ m}\right)$ and the calculation of their qualification.

Example 2 We now consider the comparison of 8 cars $(a, b, c, d, e, f, g)$ on the basis of maximum speed, volume, price and consumption. Data and aggregation procedure will not be presented here (for more details, see [2]). The related outranking relation is presented in Table 2 .

\begin{tabular}{|c|c|c|c|c|c|c|c|c|}
\hline$R$ & $a$ & $b$ & $c$ & $d$ & $e$ & $f$ & $g$ & $h$ \\
\hline$a$ & 1 & .75 & .70 & .62 & 0 & 0 & 0 & 0 \\
\hline$b$ & .76 & 1 & .90 & .82 & .82 & .82 & .82 & .80 \\
\hline$c$ & .70 & .86 & 1 & 1 & 1 & .46 & .80 & .91 \\
\hline$d$ & .64 & .65 & .94 & 1 & .88 & .22 & .94 & .74 \\
\hline$e$ & .33 & .57 & .93 & 1 & 1 & 0 & .80 & .86 \\
\hline$f$ & 0 & .73 & .64 & .92 & .76 & 1 & .96 & .80 \\
\hline$g$ & 0 & .63 & .73 & .85 & .82 & .70 & 1 & .81 \\
\hline$h$ & 0 & .60 & .64 & .60 & .77 & 0 & 0 & 1 \\
\hline
\end{tabular}

Table 2 : Outranking relation related to eight cars 
We will consider only the ordinal content of that outranking relation and we transpose the data on a $L$-scale with $c_{0}=0, c_{2 m}=1, m=27$ and $c_{m}=.5$.

The strict median-cut relation $R^{\succ m}$ corresponds to data of Table 1 . The set $C^{\text {good }}\left(G^{\succ m}\right)$ corresponds to $(\{b\},\{a, f\},\{a, g\})$ with the following qualifications:

$$
Q^{\text {good }}(\{b\})=.76, \quad Q^{\text {good }}(\{a, f\})=Q^{\text {good }}(\{a, g\})=.70 .
$$

\section{$3 \quad$ Fuzzy kernels}

A second approach to the problem of determining a good choice is to consider the valued extension of the Boolean system of equations (1).

If $\tilde{Y}()=.(\ldots, Y(x), Y(y), \ldots)$, where $\tilde{Y}(x)$ belongs to $L$ for every $x \in A$ is the characteristic vector of a fuzzy choice and indicates the credibility level of the assertion that " $x$ is part of the choice $\tilde{Y}$ ", we have to solve the following system of equations:

$$
(\tilde{Y} \circ R)(x)=\max _{y \neq x}[\min (\tilde{Y}(y), R(y, x))]=\neg \tilde{Y}(x), \quad \forall x, y \in A .
$$

The set of solutions to the system of equations (2) is called $\tilde{Y}^{\text {dom }}\left(G^{L}\right)$.

In order to compare these fuzzy solutions to the solutions obtained in $C^{\text {good }}\left(G^{L}\right)$, we define the crisp choice

$$
K_{\tilde{Y}} \subset A\left\{\begin{array}{l}
x \in K_{\tilde{Y}} \text { if } \tilde{Y}(x) \in L^{\succ m} \\
x \notin K_{\tilde{Y}} \text { otherwise }
\end{array}\right.
$$

and we consider a partial order on the elements of $\tilde{Y}^{d o m}\left(G^{L}\right): \tilde{Y}$ is sharper than $\tilde{Y}^{\prime}$, noted $\tilde{Y}^{\prime} \preceq \tilde{Y}$, iff $\forall x \in A$ : either $\tilde{Y}(x) \leq \tilde{Y}^{\prime}(x) \leq c_{m}$, either $c_{m} \leq \tilde{Y}^{\prime}(x) \leq$ $\tilde{Y}(x)$.

The subset of the sharpest solutions in $\tilde{Y}^{\text {dom }}\left(G^{L}\right)$ is called $F^{\text {dom }}\left(G^{L}\right)$.

Bisdorff and Roubens have proved that the set of crisp choices constructed from $F^{d o m}\left(G^{L}\right)$ using $(3)$ and denoted $K\left(F^{\text {dom }}\left(G^{L}\right)\right)$ coincides with $C^{\text {dom }}\left(G^{L}\right)$.

Coming back to Example 2, we obtain 3 sharpest solutions to equation (2)

$$
\begin{aligned}
\tilde{Y}_{\{b\}} & =(.24, .76, .24, .24, .24, .24, .24, .24) \\
\tilde{Y}_{\{a, f\}} & =(.70, .30, .30, .30, .30, .70, .30, .30) \\
\tilde{Y}_{\{a, g\}} & =(.70, .30, .30, .30, .30, .30, .30, .70) .
\end{aligned}
$$

In this particular case, we obtain only $Q^{\text {good }}$ and $\neg Q^{\text {good }}$ as components of the $\tilde{Y}$ 's but this is not true in general.

\section{Good and bad choices in Multi-attribute decision making}

In the framework of decision making procedures, it is often interesting to determine choice sets that correspond to bad choices. These bad choices should be ideally different from the good choices. To clarify this point, let us first consider the crisp Boolean case and define the rationality axiom of 
- Absorbance of $Y$ (see [10])

$\forall x \in \bar{Y}, \exists y \in Y, x R y=y R^{t} x$

$\bar{Y} \subseteq Y \circ R^{t}$, "the predecessors of $Y$ contain $\bar{Y}$ ".

As the stability property can be rewritten as $Y \circ R^{t} \subseteq \bar{Y}$, we immediately obtain the Boolean equation that determines the absorbent kernel (stable and absorbent choice):

$$
\bar{Y}=Y \circ R^{t} .
$$

We notice that for some digraphs (dominant) kernels and absorbent kernels may coincide (consider a digraph $G(A, R)$ with vertices $A:\{a, b, c, d\}$ and four $\operatorname{arcs}(a, b),(b, c),(c, d),(d, a) .\{a, c\}$ as well as $\{b, d\}$ are dominant and absorbent kernels or good and bad choices).

This last concept can be easily extended in the valued case. Consider the valued graph $G^{L}$ introduced in Section 2. We define the level of absorbance qualification of $Y$ as

$$
\Delta^{a b s}(Y)= \begin{cases}c_{2 m} & \text { if } Y=A \\ \min _{x \notin Y} \max _{y \in Y} R(x, y) & \text { otherwise. }\end{cases}
$$

The qualification of $Y$ being a bad choice corresponds to

$$
Q^{b a d}(Y)=\min \left(\Delta^{s t a}(Y), \Delta^{a b s}(Y)\right)>c_{m} .
$$

If $Q^{\text {bad }}(Y) \leq c_{m}, Y$ is not considered to be a bad choice.

A fuzzy absorbent kernel is a solution of equation

$$
\left(\tilde{Y} \circ R^{t}\right)(x)=\max _{y \neq x} \min \left(\tilde{Y}(y), R^{t}(y, x)\right)=\neg \tilde{Y}(x), \quad \forall x \in A .
$$

The set of solutions of equations (4) denoted $\tilde{Y}^{\text {abs }}\left(G^{L}\right)$ can be handled in the same way as done in Section 3 for $\tilde{Y}^{\text {dom }}\left(G^{L}\right)$ and creates a link between these solutions (4) and subsets of $Y$ being qualified as bad choices.

Reconsidering Example 2, we observe that $\{b\},\{c\},\{d\},\{a, e\}$ and $\{a, h\}$ are absorbent kernels in $G\left(A, R^{\succ m}\right)$. Qualification can be easily obtained and we get $Q^{\text {bad }}(\{a, c\})=.76, Q^{\text {bad }}(\{a, h\})=.74, Q^{\text {bad }}(\{c\})=.64, Q^{\text {bad }}(\{d\})=.60$, $Q^{b a d}(\{b\})=.57$.

We finally decide to keep car $b$ as the best solution noticing however that it is a bad choice. Going back to digraph $G\left(A, R^{\succ m}\right)$, we see that $b$ is at the same time dominating and dominated by all the other elements. Car $b$ is indifferent to all the other cars which is not true for $a, c, d, e, f, g, h$, since indifference is not transitive in this example.

\section{References}

[1] Bisdorff, R., Roubens, M.: On defining and computing fuzzy kernels from $L$-valued simple graphs. In: Da Ruan et al. (eds.): Intelligent Systems and Soft Computing for Nuclear science and Industry, FLINS'96 Workshop. World Scientific Publishers, Singapore (1996) 113-123 
[2] Fodor, J., Roubens, M.: Fuzzy Preference Modelling and Multi-criteria Decision Support. Kluwer Academic publishers, Dordrecht Boston London (1994)

[3] Fodor, J.C., Perny, P., Roubens, M.: Decision Making and Optimization. In: Ruspini, E., Bonissone, P., Pedrycz, W. (eds.): Handbook of Fuzzy Computation, Institute of Physics Publications and Oxford University Press, Bristol (1998) F.5.1: $1-14$

[4] Fodor, J., Orlovski S.A., Perny, P., Roubens, M.: The use of fuzzy preference models in multiple criteria: choice, ranking and sorting. In: Dubois, D., Prade, H. (eds.): Handbooks and of Fuzzy Sets, Vol. 5 (Operations Research and Statistics), Kluwer Academic Publishers, Dordrecht Boston London (1998) 69-101

[5] Kitainik, L.: Fuzzy Decision Procedures with Binary Relations: towards an unified Theory. Kluwer Academic Publishers, Dordrecht Boston London (1993)

[6] Marichal, J.-L.: Aggregation of interacting criteria by means of the discrete Choquet integral. In: Calvo,T., Mayor,G., Mesiar R. (eds.): Aggregation operators: new trends and applications. Series: Studies in Fuzziness and Soft Computing Vol. 97, Physica-Verlag, Heidelberg (2002) 224-244

[7] Perny, P., Roubens, M.: Fuzzy Relational Preference Modelling. In: Dubois, D,. and Prade, H. (eds.): Handbooks of Fuzzy Sets, Vol. 5 (Operations Research and Statistics). Kluwer Academic Publishers, Dordrecht Boston London (1998) 3-30

[8] Roy,B.: Algèbre moderne et théorie des graphes. Dunod, Paris (1969).

[9] Schmidt, G., Ströhlein, T.: Relations and Graphs; Discrete mathematics for Computer Scientists. Springer-Verlag, Berlin Heidelberg New York (1991)

[10] Schwartz, T.: The logic of Collective Choice, Columbia Univer Press, New York (1986)

[11] von Neumann, J., Morgenstern, O.: Theory of Games and Economic Behaviour. Princeton University Press, New York (1953) 